\title{
Genome-wide association study of beef bull semen attributes
}

\author{
M. L. Butler, A. R. Hartman, J. M. Bormann, R. L. Weaber, D. M. Grieger and M. M. Rolf*
}

\begin{abstract}
Background: Cattle production is dependent upon fertility because it results in producing offspring to offset production costs. A number of semen attributes are believed to affect fertility and are frequently measured as part of routine breeding soundness exams or semen collection procedures. The objective of this study was to perform a single-step genome-wide association study (ssGWAS) for beef bull semen attributes. Beef bull fertility phenotypes including volume (VOL), concentration (CONC), number of spermatozoa (NSP), initial motility (IMot), post-thaw motility (PTMot), three-hour post-thaw motility (3HRPTMot), percentage of normal spermatozoa (\%NORM), primary abnormalities (PRIM), and secondary abnormalities (SEC) were obtained from two artificial insemination (AI) centers. A total of 1819 Angus bulls with 50,624 collection records were used for ssGWAS. A five-generation pedigree was obtained from the American Angus Association and consisted of 6521 sires and 17,136 dams. Genotypes on 1163 bulls were also obtained from the American Angus Association and utilized in ssGWAS.

Results: A multi-trait animal model was used for the estimation of single nucleotide polymorphism (SNP) effects. Significant SNP were those with a $\log _{10} P$-value threshold greater than 4.0. Volume, CONC, NSP, IMot, PTMot, 3HRPTMot, \%NORM, PRIM, and SEC have five, three, six, seven, two, six, six, and two genome-wide significant SNP, respectively.

Conclusions: Several significant SNP were determined to be near or within quantitative trait loci (QTL) associated with beef bull semen attributes. In addition, genes associated with fertility were found to contain or be near the significant SNP found in the study. The results indicate there are regions of the genome that impact fertility, proving inclusion of genomic information into genetic evaluation should be advantageous for genetic improvement of male fertility traits.
\end{abstract}

\section{Background}

Fertility is a complex trait which is affected by management [1-3], nutrition $[4,5]$, and genetics $[6,7]$. The complexity of fertility may be one reason for the lack information available to beef seedstock producers for making fertility selection decisions. Other reasons may include that fertility data is not currently aggregated by beef breed associations and semen quality data from bull studs is largely proprietary. As genomic testing becomes more affordable and a part of regular management

*Correspondence: megrolf@ksu.edu

Department of Animal Science Kansas State University, Manhattan, Kansas 66502, USA practices for seedstock producers, beef cattle producers become more willing and able to utilize the technology. Utilizing genomic technology in breeding decisions allows cattle producers to more confidently incorporate young, unproven sires into their breeding programs. While using young breeding stock increases the rate of genetic change, the risk of using these bulls is higher because their expected progeny differences (EPD) accuracies are lower. Beef cattle producers do not currently have a selection tool to confidently choose more fertile bulls, nor are current versions of SNP chips enriched in regions where important variants for male fertility exist because they are largely unstudied and unidentified. 
However, thousands of semen records from beef bull semen collection facilities provide an opportunity to identify polymorphisms which may affect spermatogenesis, morphology, and motility of spermatozoa [8]. One way to truly increase the reproductive efficiency of beef cattle production is to build the capacity to identify young beef bulls with sperm abnormalities and low semen quality so that the bulls can be eliminated from the breeding population [8]. Dairy cattle research underscores the ability to capitalize on genomic technologies in these efforts, as researchers have identified multiple QTL regions [9-17] and candidate genes [18-24] associated with male and female fertility. Researchers have identified areas of the genome that are associated with fertility traits in beef bulls [25], but the number of studies is limited. Thus, the need for further validation is a necessity. The objective of this study was to perform a genomewide association study for beef bull semen attributes and to identify quantitative trait loci (QTL) regions and genes likely associated with fertility traits in beef bulls.

\section{Methods}

\section{Data collection}

Phenotypic observations for 1819 Angus bulls were obtained from two bull semen collection facilities. The phenotypic observations for beef bull semen attributes included volume (VOL), concentration (CONC), number of spermatozoa (NSP), initial motility (IMot), post-thaw motility (PTMot), three-hour post-thaw motility (3HRPTMot), percentage of normal spermatozoa (\%NORM), primary abnormalities (PRIM), and secondary abnormalities (SEC). Trait definitions are listed in Table 1. Data editing procedures were performed to ensure no VOL or CONC phenotypes were 0 , all phenotypes recorded as a percentage ranged from 0 to 100 , and all bulls had a registration number. After data editing procedures there were 50,624 total collection records utilized for the ssGWAS. Bull stud A contributed 48,131 collection records from 1570 bulls and bull stud B contributed an additional 2493 records from 256 bulls. Summary statistics are shown in Table 1.

The American Angus Association provided pedigree information for 1819 bulls and SNP data for 1163 bulls. The pedigree contained 6521 sires and 17,136 dams. The maximum number of SNP per bull was 54,609 . Data were edited to remove SNP with a call rate of $<0.90(n=3921)$ and minor allele frequency of $<0.05(n=13,478)$. Animals with a call rate of $<0.90$ were removed $(n=3)$. Imputation was performed by genotyping providers to the American Angus Association, so that all genotyped bulls had 54,609 SNP. After quality control filtering, a total of 38,515 SNP from 1160 animals were available for analysis. The American Angus Association also provided the SNP positions

Table 1 Trait list, definitions, and summary statistics of beef bull fertility traits collected at an artificial insemination. All post-thaw motility measures are observed the day after the sample is collected. All traits are measured within one hour except for three-hour post-thaw motility

\begin{tabular}{|c|c|c|c|c|c|c|c|}
\hline Trait & Units & Definition & $\mathbf{N}$ & Mean & Std Dev & Minimum & Maximum \\
\hline Volume (VOL) & $\mathrm{mL}$ & $\begin{array}{l}\text { total amount of the ejaculate, measured } \\
\text { by milliliters }\end{array}$ & 44,431 & 7.96 & 4.22 & 0.10 & 74.00 \\
\hline Concentration (CONC) & Million/mL & $\begin{array}{l}\text { relative amount of sperm cells per ejacu- } \\
\text { late, measured by a colorimeter }\end{array}$ & 44,038 & 1021.16 & 495.02 & 10.00 & 3906.00 \\
\hline Number of spermatozoa (NSP) & Million & $\begin{array}{l}\text { calculated by multiplying sperm } \\
\text { concentration and semen volume; } \\
\text { expressed in millions }\end{array}$ & 44,418 & 49.18 & 16.16 & 0.00 & 100.00 \\
\hline Initial Motility (IMot) ${ }^{a}$ & $\%$ & $\begin{array}{l}\text { percentage of progressively swimming } \\
\text { spermatozoa in the ejaculate immedi- } \\
\text { ately after collection }\end{array}$ & 44,038 & 8004.18 & 5519.61 & 64.50 & $69,795.00$ \\
\hline Post-thaw Motility (PTMot) ${ }^{a}$ & $\%$ & $\begin{array}{l}\text { percentage of progressively swimming } \\
\text { spermatozoa in the ejaculate, measured } \\
\text { within one hour of thawing }\end{array}$ & 29,877 & 43.53 & 13.78 & 0.00 & 75.00 \\
\hline $\begin{array}{l}\text { Three-hour post-thaw motility } \\
\text { (3HRPTMot) }^{\text {a }}\end{array}$ & $\%$ & $\begin{array}{l}\text { percentage of progressively swimming } \\
\text { spermatozoa in the ejaculate, measured } \\
\text { three hours after thawing }\end{array}$ & 8299 & 15.72 & 12.48 & 0.00 & 60.00 \\
\hline $\begin{array}{l}\text { Percentage of Normal Spermatozoa } \\
(\% \text { NORM })^{\mathrm{a}}\end{array}$ & $\%$ & $\begin{array}{l}\text { percent morphologically normal sper- } \\
\text { matozoa }\end{array}$ & 19,455 & 75.18 & 8.37 & 2.00 & 100.00 \\
\hline Primary Abnormalities (PRIM) ${ }^{\mathrm{a}}$ & $\%$ & $\begin{array}{l}\text { percentage of spermatozoa with a } \\
\text { defect to the head }\end{array}$ & 19,452 & 13.00 & 7.53 & 0.00 & 100.00 \\
\hline Secondary Abnormalities (SEC) ${ }^{a}$ & $\%$ & $\begin{array}{l}\text { percentage of spermatozoa with a } \\
\text { defect to the tail }\end{array}$ & 19,521 & 12.09 & 7.70 & 0.00 & 100.00 \\
\hline
\end{tabular}

${ }^{a}$ Trait is measured subjectively by a trained laboratory technician 
and the reference genome used by the American Angus Association was UMD3.1.

\section{Statistical analysis}

Beef bull fertility is known to be affected by a variety of environmental factors. A detailed model selection procedure was utilized to select the fixed effects and covariates to include in the analysis and calculate the variance components as described by [26]. In brief, we used a forward selection procedure to identify factors that significantly affected beef bull semen attributes. The factors included in the final model included location where the bull was collected (Location), the class effect of day within year (DayYear), a covariate for age of the bull on the day of collection in days (Age), a covariate for days since previous collection (DaysSince), and a covariate effect of cumulative comprehensive climate index (cumCCI) over the spermatogenesis cycle. The mathematical model used for analysis was as follows:

$$
y_{i j k}=S_{j}+W_{p e}+\text { Location }+ \text { DayYear }+ \text { Age }+ \text { DaysSince }+ \text { Cum CCI }+\varepsilon
$$

where $y$ is the phenotype being evaluated, $S_{j}$ is a random animal effect, $W_{\text {pe }}$ is the permanent environment effect to account for repeated measures, and $\varepsilon$ is the residual.

The mixed model used in the current study is:

$$
\left[\begin{array}{ccc}
X^{\prime} X & X^{\prime} Z_{a} & X^{\prime} W_{p e} \\
X^{\prime} Z_{a} & Z_{a}^{\prime} Z_{a}+\lambda H^{-1} & Z_{a}^{\prime} W_{p e} \\
X^{\prime} W_{p e} & Z_{a}^{\prime} W_{p e} & W_{p e}^{\prime} W_{p e}+I \alpha_{p e}
\end{array}\right]\left[\begin{array}{c}
\hat{b} \\
\hat{a} \\
\hat{p e}
\end{array}\right]=\left[\begin{array}{c}
X^{\prime} y \\
Z^{\prime} y \\
W^{\prime} y
\end{array}\right]
$$

where $\mathrm{X}$ is an incidence matrix relating the phenotypic observations to the fixed effects in the model (detailed above), $\mathrm{Z}_{\mathrm{a}}$ is an incidence matrix relating the phenotypic observations to the additive direct genetic effects, $W_{\text {pe }}$ is an incidence matrix relating the phenotypic observations to the additive permanent environment genetic effects, $\lambda$ is the ratio of residual and additive direct genetic variance, $\mathrm{I}$ is an identity matrix, and $\alpha$ is the ratio of residual variance and additive permanent environment variance, $\hat{\boldsymbol{b}}$ is a vector of the fixed effect solutions, $\hat{\boldsymbol{a}}$ is a vector of additive direct genetic effects, $\hat{\boldsymbol{p} e}$ is a vector of additive permanent environment genetic effects, and $y$ is a vector of phenotypic observations. Estimates for specific variance components are found in [26].

All bulls in this study had phenotypic data but because not all phenotyped animals were genotyped, and to include all available information into the analysis, both pedigree and genomic information were utilized in the genetic evaluation of beef bull fertility using a singlestep genomic best linear unbiased prediction (ssGBLUP) model. In a ssGBLUP model, the numerator relationship matrix (A) is replaced with $\mathrm{H}$, which augments A with the genotype information. The inverse of the $\mathrm{H}$ matrix is generated as follows:

$$
\mathbf{H}^{-1}=\mathbf{A}^{-1}+\left[\begin{array}{lc}
\mathbf{0} & \mathbf{0} \\
\mathbf{0} & \mathbf{G}^{-1}-\mathbf{A}_{22}^{-1}
\end{array}\right]
$$

where $\mathrm{A}^{-1}$ is the inverse of the pedigree-based numerator relationship matrix, $\mathrm{A}^{-1}{ }_{22}$ is a subset of the numerator relationship matrix for the genotyped individuals, and $\mathrm{G}$ is the genomic relationship matrix for the genotyped individuals [27].

The following algorithm was used to back solve for the SNP effects for the ssGWAS [28]:

$$
a=Z u
$$

where $\mathbf{a}$ is a vector of breeding values for the genotyped individuals generated from BLUPF90, $\mathbf{Z}$ is a matrix relating individuals to phenotypes, and $\mathbf{u}$ is a vector of SNP marker effects.

The variance for the genotyped animal effects is as follows [28]:

$$
\operatorname{var}(\boldsymbol{a})=\operatorname{var}(\boldsymbol{Z u})=\boldsymbol{G} \sigma_{a}^{2}=\boldsymbol{Z I} \boldsymbol{Z}^{\prime} \lambda
$$

where $\mathbf{G}$ is the genomic relationship matrix, $\mathbf{I}$ is an identity matrix, and $\boldsymbol{\lambda}$ is the ratio of the SNP marker effect variance and the breeding value variance.

The SNP effects were predicted utilizing the following equation:

$$
\hat{\boldsymbol{u}}=\boldsymbol{I} \boldsymbol{Z}^{\prime}\left(\boldsymbol{Z} \boldsymbol{I} \boldsymbol{Z}^{\prime}\right)^{-1} \hat{\boldsymbol{a}}
$$

where $\mathbf{u}$ is a vector of SNP marker effects, $\mathbf{I}$ is an identity matrix, $\mathbf{Z}$ is a matrix relating individuals to phenotypes, and $\mathbf{a}$ is a vector of breeding values for the genotyped individuals. Each SNP was assumed to have an equal allele substitution effect variance, and it was assumed the SNP affects followed the infinitesimal model. Thus, an unweighted ssGBLUP was performed.

SNP effects were obtained from one trivariate and six bivariate analyses. VOL, CONC, and NSP were included in a trivariate analysis. Due to convergence issues, bivariate analyses were performed among the remaining groups of traits. Initial motility, PTMot, and 3HRPTMot SNP effects were generated from three different bivariate analyses, and \%NORM, PRIM, and SEC from an additional three bivariate analyses. For traits where a bivariate analysis was performed, significant SNP from both bivariate analyses are reported. Utilizing multi-trait analyses allows for multiple, genetically correlated traits to be included in the model and improves the predicative capability for each trait. The $P$-values associated with the SNP effects were obtained from the POSTGSF90 program 
within the BLUPF90 software suite as detailed in [29]. The $p$-value for the SNP effect is obtained by [30]:

$$
p_{i}=2\left(1-\Phi\left(\left|\frac{\alpha_{i}}{s d\left(a_{i}\right)}\right|\right)\right)
$$

where $\alpha_{i}$ is the estimate of the marker effects, sd is the standard deviation, and $\Phi$ is the cumulative standard normal function.

The $P$-values are generated by back solving for SNP effects from the breeding value estimates. This approach is possible because the fitting of animal as a random effect to generate breeding value estimates is an equivalent model to fitting all SNPs as random effects and solving for these effects directly [31].

After obtaining the SNP effects $(\widehat{\boldsymbol{u}})$ and their $P$-values, Manhattan plots for all nine traits were generated utilizing the CMplot package in $\mathrm{R}$ [32]. Utilizing the Qvalue $\mathrm{R}$ package [33] the $-\log _{10} P$-values were converted to $p$-values so that the false discovery rate (FDR) could be calculated for each $P$-value. While there were no significant SNP identified at an FDR threshold of $<0.0001,<0.001$, $<0.01,<0.025$, or $<0.05$, several significant SNP existed with a $-\log _{10} P$-value threshold of 4.0 (Table 2). This less stringent threshold was chosen to allow investigation of the potential biological significance of the QTL regions identified, and is not detrimental to efforts to utilize these results in an unweighted ssGBLUP that does not specifically fit the effect of any single SNP for genomic prediction.

\section{QTL analysis}

To account for linkage disequilibrium in the Bos taurus genome, QTL regions were formed 250,000 kilobases upstream and downstream from the significant SNP locations [34]. The regions were utilized to identify previously reported QTL which were near the significant SNP ( $\pm 250,000$ kilobases). The QTL regions were identified utilizing the cattle QTL database [35]. For each trait, previously reported QTL were identified close to significant SNP. Where possible, SNP were identified by the corresponding rs number. If no rs number was available, the SNP name was utilized. In addition, the same QTL region boundaries were used to identify genes near the significant SNP using the National Center of Biotechnology Information (NCBI) database. Putative candidate genes were identified near significant SNP and examined for biological meaning. The genes specifically associated with beef bull semen attributes were identified based on the gene functions [36]. The gene list was then used to perform a functional annotation analysis utilizing the Database for Annotation, Visualization, and Integrated
Discovery (DAVID 6.8) [37, 38] for each individual beef bull semen attribute.

\section{Results and discussion}

Significant SNP were identified for all beef bull semen attributes. Significant SNP are reported in Table 2 and Manhattan plots are provided in Figs. 1, 2, and 3. For each trait, previously reported QTL were identified close to significant SNP (Table 3). Putative candidate genes specifically associated with beef bull semen attributes are outlined in Table 4.

For VOL, five strongly associated SNP were identified, including BTB-01549373, rs41666488, rs109736826, rs109268478, and rs41575945 (Table 2). Three QTL regions associated with conception rate or non-return rate in dairy cattle $[10,39,52]$ were near BTB-01549373 and rs41575945 (Table 3). While conception and nonreturn rate are generally associated with female fertility, it is interesting that SNP significantly associated with male fertility traits are also near these QTL regions. This is evidence that the ability to successfully reproduce is dependent not only on the female, but also on the semen quantity and quality of the bull [52]. This may also indicate that although the few reported genetic correlations between male and female fertility are low [47, 53, 54], there may be some pleiotropy whereby mutations control aspects of both male and female fertility.

Three significant SNP were identified for CONC, which included rs43067163, rs41623602, and rs29023737 (Table 2). A previously reported mature height QTL region was close to rs41623602 [46]. In addition, a previously reported QTL region associated with weaning weight and mature height was in proximity to rs41623602 (Table 3) [46]. While there are few studies that directly relate size of the bull to the semen quality, weight and height are generally associated with maturity, and the association could be indicative that more mature, larger bulls have greater semen production. In the current study, weight and hip height of the bull at the time of collection did not contribute significantly to concentration [26].

Seven SNP had significant associations with NSP (Table 2). Three different previously reported conception rate QTL regions $[39,40]$ neighbored significant SNP for NSP. In addition, previously reported QTL regions associated with non-return rate were in the vicinity of two significant SNP from the current study [11, 41]. Like conception rate, non-return rate is usually related to female fertility, but this association provides further validation that male fertility traits are integral in the reproductive success of the herd [52] and/or that male and female fertility share a genetic correlation due to pleiotropy in these regions of the genome. 
Table 2 Genomic regions identified by genome-wide association study contributing significantly to beef bull semen attributes. Significant SNP with a $p$-value of $<0.00001$

\begin{tabular}{|c|c|c|c|c|c|}
\hline Trait & SNP Name & $-\log _{10} p$-value & rsID & Chromosome & Position \\
\hline \multirow[t]{5}{*}{ Volume } & BTB-01549373 & 4.01 & No rsID & 2 & $81,679,350$ \\
\hline & Hapmap48133-BTA-96243 & 4.21 & rs41666488 & 3 & $61,947,686$ \\
\hline & ARS-BFGL-NGS-71827 & 4.26 & rs109736826 & 3 & $112,997,892$ \\
\hline & ARS-BFGL-NGS-25127 & 4.19 & rs109268478 & 6 & $102,859,342$ \\
\hline & Hapmap49899-BTA-18490 & 4.44 & rs41575945 & 27 & $3,495,048$ \\
\hline \multirow[t]{3}{*}{ Concentration } & BTB-01963898 & 4.03 & rs43067163 & 1 & $133,936,071$ \\
\hline & BTA-122016-no-rs & 4.49 & rs41623602 & 3 & $38,811,314$ \\
\hline & Hapmap55203-rs29023737 & 4.12 & rs29023737 & 5 & $3,645,270$ \\
\hline \multirow[t]{7}{*}{ Number of Spermatozoa } & BTA-110980-no-rs & 4.83 & rs41618035 & 1 & $31,173,269$ \\
\hline & ARS-BFGL-NGS-101891 & 4.28 & rs109740774 & 6 & $75,137,290$ \\
\hline & Hapmap33368-BTA-146079 & 4.25 & rs43567728 & 8 & $89,743,053$ \\
\hline & Hapmap44146-BTA-83959 & 4.07 & rs41661101 & 9 & $50,922,485$ \\
\hline & ARS-BFGL-NGS-112914 & 4.71 & rs110005257 & 11 & $99,686,154$ \\
\hline & ARS-BFGL-NGS-101386 & 4.55 & rs110190516 & 17 & $67,017,094$ \\
\hline & ARS-BFGL-NGS-85970 & 4.04 & rs108993490 & 24 & $16,118,203$ \\
\hline \multirow[t]{6}{*}{ Initial Motility } & Hapmap48211-BTA-120784 & 4.69 & rs41623436 & 1 & $152,412,536$ \\
\hline & ARS-BFGL-NGS-74920 & 4.54 & rs109798673 & 2 & 827,626 \\
\hline & BTB-00319289 & 4.13 & rs43526428 & 7 & $73,777,772$ \\
\hline & Hapmap59148-ss46527122 & 4.10 & rs29003479 & 11 & $63,430,172$ \\
\hline & BTB-01751684 & 4.04 & rs42861585 & 23 & $46,295,105$ \\
\hline & Hapmap23061-BTC-074055 & 4.05 & rs109512383 & 25 & $30,964,321$ \\
\hline \multirow[t]{7}{*}{ Post-thaw Motility } & Hapmap48211-BTA-120784 & 4.75 & rs41623436 & 1 & $152,412,536$ \\
\hline & ARS-BFGL-NGS-74920 & 5.63 & rs109798673 & 2 & 827,626 \\
\hline & Hapmap45703-BTA-105835 & 4.10 & rs41611445 & 2 & $82,083,660$ \\
\hline & BTB-01323835 & 4.22 & rs42446055 & 4 & $89,708,810$ \\
\hline & BTB-01537954 & 4.32 & rs42653645 & 11 & $25,036,830$ \\
\hline & ARS-BFGL-NGS-41002 & 4.52 & rs110418161 & 16 & $36,415,574$ \\
\hline & ARS-BFGL-NGS-111058 & 4.82 & rs109719529 & 17 & $56,344,620$ \\
\hline \multirow[t]{2}{*}{ Three Hour Post-thaw Motility } & Hapmap59148-ss46527122 & 4.50 & rs29003479 & 11 & $63,430,172$ \\
\hline & ARS-BFGL-NGS-111058 & 4.49 & rs109719529 & 17 & $56,344,620$ \\
\hline \multirow[t]{6}{*}{ Percentage of Normal Spermatozoa } & BTA-50285-no-rs & 4.41 & rs41606310 & 1 & $7,669,386$ \\
\hline & ARS-BFGL-NGS-43775 & 5.94 & rs110964837 & 2 & $73,209,337$ \\
\hline & BTA-91078-no-rs & 4.85 & rs41594758 & 3 & $43,031,873$ \\
\hline & ARS-BFGL-NGS-25359 & 4.71 & rs109928164 & 5 & $70,049,126$ \\
\hline & BTA-74480-no-rs & 4.66 & rs41591913 & 5 & $84,578,337$ \\
\hline & BTA-98744-no-rs & 4.68 & rs41666416 & 10 & $68,089,016$ \\
\hline \multirow[t]{6}{*}{ Primary Abnormalities } & BTA-115758-no-rs & 4.11 & rs41566683 & 1 & $36,903,496$ \\
\hline & Hapmap57078-ss46526391 & 4.95 & rs41255529 & 1 & $60,956,897$ \\
\hline & ARS-BFGL-NGS-44433 & 4.22 & rs110487590 & 1 & $63,799,085$ \\
\hline & BTB-01831345 & 4.51 & rs42940192 & 4 & $10,648,384$ \\
\hline & ARS-BFGL-NGS-80666 & 4.05 & rs110425782 & 20 & $68,324,872$ \\
\hline & Hapmap42996-BTA-60916 & 4.16 & rs41646489 & 22 & $33,690,494$ \\
\hline Secondary Abnormalities & Hapmap42996-BTA-60916 & 4.28 & rs41646489 & 22 & $33,690,494$ \\
\hline
\end{tabular}

For IMot, 6 SNP on 6 different chromosomes were significant, including rs41623436, rs109798673, rs43526428, rs29003479, rs42861585, and rs109512383. One SNP, rs29003479, on chromosome 11 was significant in both bivariate analyses for initial motility and the other five were only significant in one of the analyses. The SNP significant in both bivariate analyses was near a previously reported scrotal circumference QTL region [46]. Scrotal 


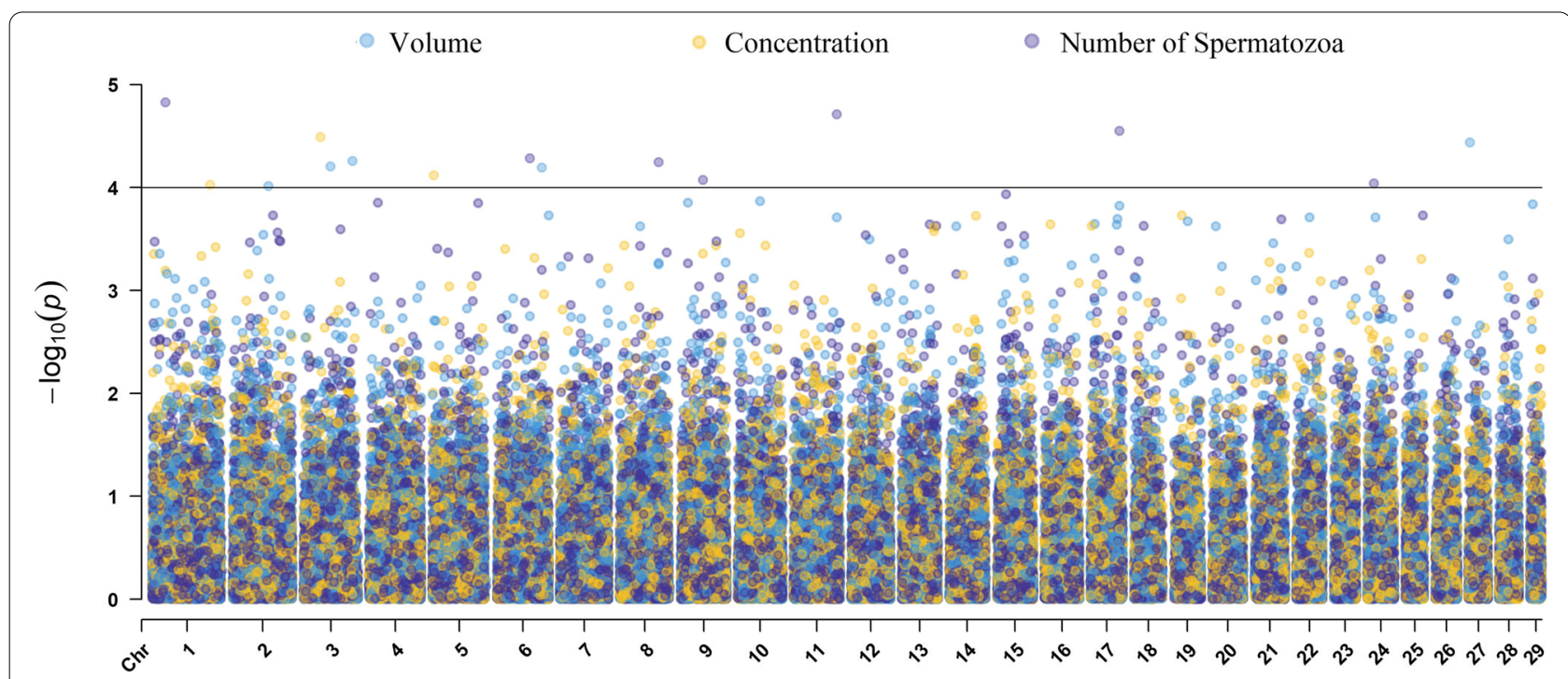

Fig. 1 Manhattan plot showing the result of genome-wide association mapping for volume, concentration, and number of spermatozoa with a significance threshold of 4.0. A trivariate analysis was performed
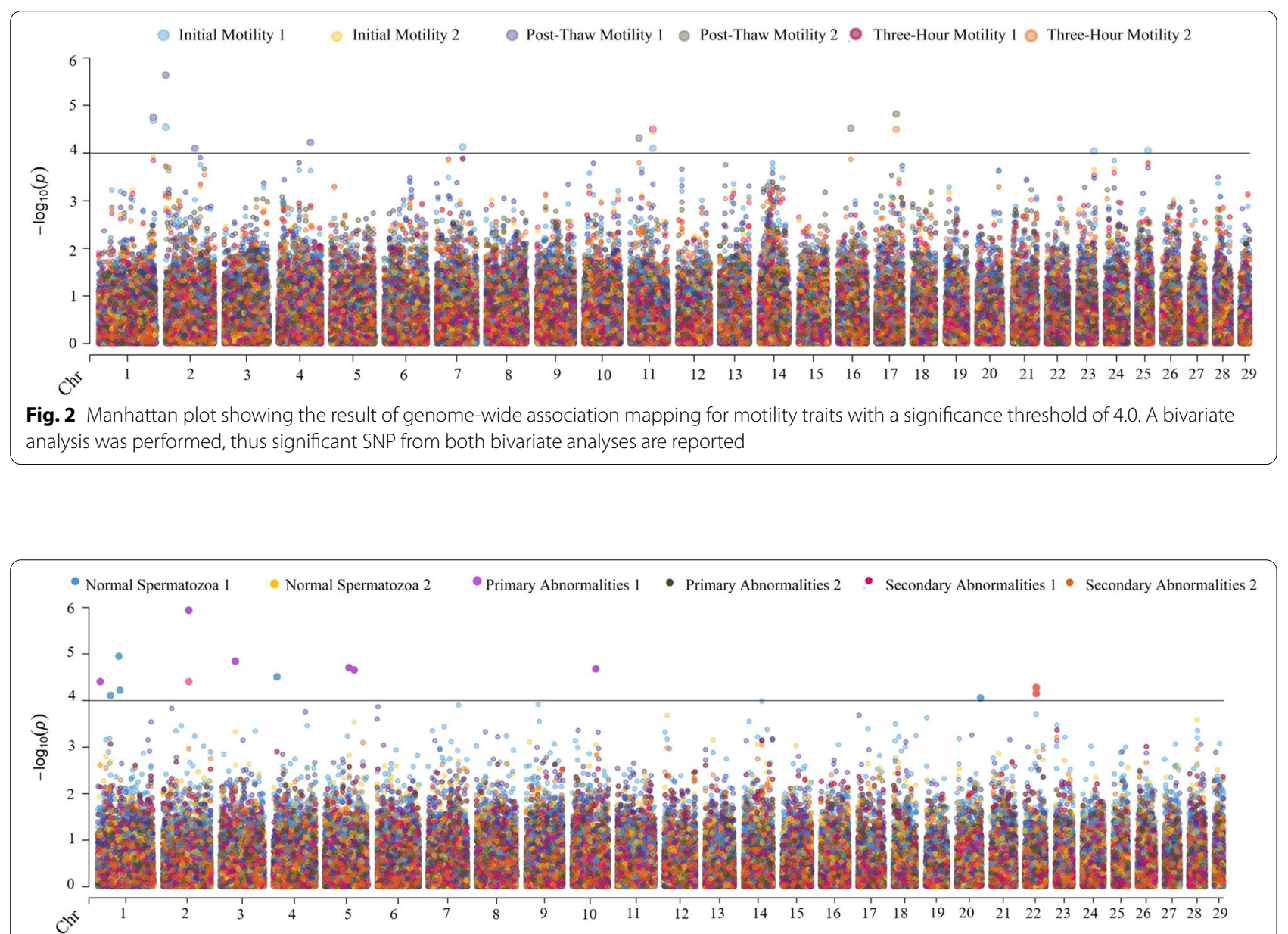

Fig. 3 Manhattan plot showing the result of genome-wide association mapping for percentage of normal spermatozoa and abnormality traits with a significance threshold of 4.0. A bivariate analysis was performed, thus significant SNP from both bivariate analyses are reported 
Table 3 Previously reported fertility-associated QTL regions which overlap significant SNP identified for beef bull semen attributes in this study

\begin{tabular}{|c|c|c|c|c|c|}
\hline Trait & SNP Name & rsID & Chr:Position & QTL Trait & Source \\
\hline \multirow[t]{3}{*}{ Volume } & BTB-01549373 & No rsID & 2: 81679350 & Conception rate & [39] \\
\hline & & & & Non-return rate & {$[9]$} \\
\hline & Hapmap49899-BTA-18490 & rs41575945 & $27: 3495048$ & Non-return rate & [10] \\
\hline \multirow[t]{10}{*}{ Number of Spermatozoa } & BTA-110980-no-rs & rs41618035 & 1:31173269 & Conception rate & [40] \\
\hline & & & & Inseminations per conception & [11] \\
\hline & & & & Non-return rate & [11] \\
\hline & ARS-BFGL-NGS-101891 & rs109740774 & $6: 75137290$ & First service conception & [39] \\
\hline & Hapmap33368-BTA-146079 & rs43567728 & 8: 89743053 & Conception rate & [39] \\
\hline & Hapmap44146-BTA-83959 & rs41661101 & 9:50922485 & Non-return rate & [41] \\
\hline & & & & $\begin{array}{l}\text { Structural soundness of feet, } \\
\text { legs, penis, and prepuce }\end{array}$ & [42] \\
\hline & ARS-BFGL-NGS-101386 & rs110190516 & 17: 67017094 & Non-return rate & [43] \\
\hline & ARS-BFGL-NGS-85970 & rs108993490 & 24:16118203 & First service conception rate & [39] \\
\hline & & & & Conception rate & [39] \\
\hline \multirow[t]{10}{*}{ Initial Motility } & ARS-BFGL-NGS-74920 & rs 109798673 & $2: 827626$ & Conception rate & [39] \\
\hline & & & & Fertilization rate & {$[38]$} \\
\hline & BTB-00319289 & rs43526428 & 7:73777772 & Conception rate & {$[39,44]$} \\
\hline & & & & Daughter pregnancy rate & {$[21,44]$} \\
\hline & & & & Sire conception rate & {$[45]$} \\
\hline & & & & Sexual precocity & [45] \\
\hline & Hapmap59148-ss46527122 & rs 29003479 & $11: 63430172$ & Scrotal circumference & [46] \\
\hline & Hapmap23061-BTC-074055 & rs109512383 & 25: 30964321 & Conception rate & [39] \\
\hline & & & & Scrotal circumference & {$[46]$} \\
\hline & & & & Sperm average path velocity & [12] \\
\hline \multirow[t]{5}{*}{ Post-thaw Motility } & ARS-BFGL-NGS-74920 & rs109798673 & $2: 827626$ & Conception Rate & [39] \\
\hline & & & & Fertilization Rate & [38] \\
\hline & Hapmap45703-BTA-105835 & rs41611445 & 2: 82083660 & Conception rate & [39] \\
\hline & & & & Non-return rate & [9] \\
\hline & BTB-01537954 & rs42653645 & $11: 25036830$ & Sperm motility & {$[12,16]$} \\
\hline Three-hour Post-thaw Motility & Hapmap59148-ss46527122 & rs29003479 & $11: 63430172$ & Scrotal circumference & {$[46]$} \\
\hline \multirow[t]{7}{*}{ Percentage of Normal Spermatozoa } & BTA-50285-no-rs & rs41606310 & 1: 7669386 & Daughter pregnancy rate & {$[22]$} \\
\hline & ARS-BFGL-NGS-43775 & rs110964837 & 2:73209337 & Conception rate & $\begin{array}{l}{[12,17,} \\
39]\end{array}$ \\
\hline & & & & Non-return rate & [9] \\
\hline & ARS-BFGL-NGS-25359 & rs109928164 & 5: 70049126 & Daughter pregnancy rate & [17] \\
\hline & BTA-74480-no-rs & rs41591913 & $5: 84578337$ & Conception Rate & [17] \\
\hline & BTA-98744-no-rs & rs41666416 & 10: 68089016 & Fertility index & [23] \\
\hline & & & & Scrotal circumference & [47] \\
\hline
\end{tabular}


Table 3 (continued)

\begin{tabular}{|c|c|c|c|c|c|}
\hline Trait & SNP Name & rsID & Chr:Position & QTL Trait & Source \\
\hline \multirow[t]{12}{*}{ Primary Abnormalities } & BTA-115758-no-rs & rs41566683 & 1: 36903496 & Conception rate & {$[39,40]$} \\
\hline & Hapmap57078-ss46526391 & rs41255529 & $1: 60956897$ & Conception rate & {$[39,40]$} \\
\hline & & & & First service conception & [39] \\
\hline & & & & Non-return rate & {$[9]$} \\
\hline & & & & Scrotal circumference & {$[46]$} \\
\hline & ARS-BFGL-NGS-44433 & rs110487590 & 1:63799085 & Conception rate & {$[17,40]$} \\
\hline & & & & Daughter pregnancy rate & {$[17]$} \\
\hline & & & & Non-return rate & {$[9]$} \\
\hline & & & & Scrotal circumference & {$[46]$} \\
\hline & & & & Testicular hypoplasia & {$[48]$} \\
\hline & BTB-01831345 & rs42940192 & 4:10648384 & Non-return rate & {$[49,50]$} \\
\hline & & & & Male fertility & {$[51]$} \\
\hline
\end{tabular}

Table 4 Significant SNP with putative candidate genes for semen production traits

\begin{tabular}{|c|c|c|c|c|c|c|}
\hline SNP & Trait & Chromosome: Pos & Closest gene & Biological Process & $\begin{array}{l}\text { Distance } \\
\text { from gene } \\
\text { (bp) }\end{array}$ & $\begin{array}{l}\text { Relative } \\
\text { Location of SNP } \\
\text { to gene }\end{array}$ \\
\hline \multirow[t]{4}{*}{ rs109798673 } & Initial Motility & $2: 827626$ & HERC2 & Causes reduced spermatozoa motility & 0 & Within \\
\hline & Initial Motility & $2: 827626$ & OCA2 & $\begin{array}{l}\text { Contributes to spermatid develop- } \\
\text { ment }\end{array}$ & 196,400 & Downstream \\
\hline & Post-thaw Motility & $2: 827626$ & HERC2 & $\begin{array}{l}\text { Caused reduced spermatozoa motil- } \\
\text { ity }\end{array}$ & 0 & Within \\
\hline & Post-thaw Motility & $2: 827626$ & OCA2 & $\begin{array}{l}\text { Contributes to spermatid develop- } \\
\text { ment }\end{array}$ & 196,400 & Downstream \\
\hline rs110425782 & Primary Abnormalities & 20:68324872 & LOC101902976 & Necessity for sperm motility & 108,733 & Downstream \\
\hline
\end{tabular}

circumference has been reported to be strongly and positively genetically correlated with motility $[6,55]$. A previously reported QTL for sperm average path velocity overlapped the QTL region for rs109512383 [12]. Sperm average path velocity is a measure obtained from a computer-assisted semen analysis and represents the average trajectory of the sperm cell. The trajectory of the spermatozoa is dependent upon the ability of the spermatozoa to move, thus providing further evidence of the relationship between initial motility and sperm average path velocity. A QTL region for sire conception rate [45] was in the vicinity of rs43526428, a SNP significant for IMot (Table 3). In addition, a reported conception rate QTL region $[39,56]$, and in the current study, a significant SNP for IMot was close to this region. Once the spermatozoa are deposited into the female reproductive tract, it is essential for the sperm cells to progress towards the oocyte for fertilization and, therefore conception [44]. Interestingly, a QTL region found to influence sexual precocity [45] is near a significant SNP associated with IMot in this study. This is interesting because the average age of bulls in this study was just over two years old, with the youngest bull in the evaluation just under a year old. Bull studs often collect very young bulls and producers demand the semen because these bulls often have more desirable EPD (albeit unproven and lower accuracy) than their older stud mates. Young bulls must have the libido and semen quality to produce viable sperm, so the relationship between sexual precocity and initial motility could indicate young bulls are able to produce a viable ejaculate. Quantitative trait loci associated with milk composition $[13,14]$ and interval to first estrus after calving [15] were found neighboring rs41623436. A recent study [25] found the same results with crossbred beef bulls, where a significant SNP window was within a QTL for interval to first estrus after calving. Return to estrus after calving is dependent on the hormones gonadotropin releasing hormone $(\mathrm{GnRH})$, luteinizing hormone (LH), and follicle stimulating hormone (FSH). The production of spermatozoa is also dependent on $\mathrm{GnRH}$, signaling 
the release of LH to induce the Leydig cells to produce testosterone. Testosterone paired with FSH signaling to the Sertoli cells causes the production of male gametes. It could be speculated that females which return to estrus more quickly after calving have increased hormone signaling, which could indicate males which have significant SNP near these QTL additionally have stronger hormone signaling; and therefore; produce more and higher quality gametes.

Seven SNP were identified for PTMot (Table 3). A QTL region on chromosome 11 which reportedly influences spermatozoa motility $[12,16]$ encompassed rs42653645, a SNP significant for PTMot. Two different conception rate QTL regions have been reported [39] near two SNP significant for PTMot in this study. As motile spermatozoa are necessary for conception, this is a logical relationship.

Six significant SNP were identified for \%NORM (Table 2). The SNP were rs41606310 on chromosome one, rs110964837 on chromosome two, rs41594758 on chromosome three, rs109928164 on chromosome five, rs41591913 on chromosome five, and rs41666416 on chromosome ten. Notably, rs110964837 was significant in both bivariate models where percentage of normal spermatozoa was evaluated with either PRIM or SEC. The SNP rs110964837 was one of the two SNP near conception-rate QTL regions and a non-return rate QTL region $[9,12,17,39]$. Previously reported scrotal circumference QTL regions [46], and a significant SNP for \%NORM in the current study was near the reported region. Two conception-rate QTL regions and a non-return rate QTL region [9, 12, 17, 39] were close to SNP significantly associated with \%NORM. As previously discussed, SNP significantly associated with male fertility traits nearby fertility-related QTL regions is further evidence that the quality of a bull's semen is important for conception or that the same genes underlie attributes affecting conception in both males and females.

Primary abnormalities had six strongly associated SNP: rs41566683, rs41255529, rs110487590, rs42940192, rs110425782, and rs41646489 (Table 2). A previously reported scrotal circumference QTL region found in a population of Angus bulls [46] was near rs41255529, a SNP significant for PRIM. Previously reported genetic correlations between scrotal circumference and PRIM indicate increased scrotal circumference results in fewer PRIM $[41,44]$. Three significant SNP for PRIM were in proximity to QTL regions associated with conception rate and non-return rate $[39,40,46]$. Primary abnormalities are abnormalities of the head, and without proper head formation, the sperm cell cannot penetrate the zona pellucida on the oocyte and cause conception [44].
All genes near SNP significant for IMot are outlined in Table 4. A SNP contributing significantly to IMot was within the gene E3 ubiquitin protein ligase 2 (HERC2). In addition, the significant SNP within HERC2 was also downstream of the gene melanosomal transmembrane protein (OCA2). The gene OCA2 is most commonly associated with albinism; however, it also contributes to spermatid development [36]. Reported in Table 4 are additional genes identified within the QTL regions for IMot.

A SNP significant for PTMot was in proximity to collagen like tail subunit of asymmetric acetylcholinesterase (COLQ), which contributes to the structural integrity of the extracellular matrix and heparin binding [57]. It is important to note heparin is an important enhancer of capacitation of the bovine spermatozoa [19]. Furthermore, heparin-binding proteins allow the spermatozoa to undergo the acrosome reaction [20]. In addition, a significant SNP for PTMot was in proximity to the heat shock protein family B (small) member 8 (HSPB8) protein. Elevated heat shock protein levels have been speculated to be associated with immature spermatozoa [58]. This research addressed human infertility and found that an increase in the $70-\mathrm{kDa}$ heat shock protein HspA2 resulted in spermatozoa not completing the phases of changing the plasma membrane during epididymal maturation and having larger amounts of cytoplasmic proteins in mature spermatozoa [58]. As previously discussed, heat shock protein can affect the maturation of the spermatozoa and cause infertility [58]. Reported in Table 4 are additional genes identified near SNP affecting PTMot.

Although not within $\pm 250,000$ kilobases of the significant SNP, a pertinent gene nearby was the major facilitator superfamily domain containing 14A (MFSD14A) gene. Also known as HIAT1, it was 300,000 kilobases upstream of the SNP. The MFSD14A gene is important for spermatid nucleus differentiation and sperm mitochondrion organization. It has been reported that mice with a disruption to the MFSD14A gene are infertile due to incomplete acrosome formation and round head defects [59].

Primary abnormalities had a significant SNP in the vicinity of a gene on chromosome 20 (Table 4). Primary abnormalities had a significant SNP within sperm mitochondrial-associated cysteine-rich protein-like (LOC101902976). Sperm-mitochondrial cysteine-rich protein is located in the mitochondrial capsule and plays a role in sperm motility [42]. While the mitochondria are located in the midpiece of the spermatozoa rather than the head, it is still important to note it is associated with male fertility. 


\section{Conclusion}

Several QTL associated with fertility traits were identified in this study and validated by previously published literature. In addition, genes within the QTL regions for beef bull semen attributes were discussed. While a few gene functions could be associated with beef bull semen attributes, some of the genes found in the current study do not have any known association with fertility traits reported in previous literature and have unknown related physiological association. Results from the current study, paired with findings in previous literature, validate that bull fertility traits are controlled by genetic factors. Identification of the QTL regions and SNP positions associated with these traits provides the knowledge necessary to enrich these regions in future iterations of SNP chips used within the cattle industries, and thus increase genomic prediction accuracies for these traits should the industry add them to routine genetic evaluation. The increased use of genomic testing in tandem with the number of phenotypes routinely recorded by bull semen collection facilities makes identification of beef bulls with superior genetic merit for fertility traits feasible. This advancement would increase profitability of beef producers by improving fertilization rate, increasing overall herd reproductive rate, and providing producers another genetic tool to use in making selection decisions.

\section{Acknowledgements}

We thank the bull stud collection facilities for providing the phenotypes for the genetic evaluation. Appreciation is expressed to the American Angus Association and its members for providing the pedigree and genotyping information. Finally, we wish to thank Daniela Lourenco from the University of Georgia for her troubleshooting assistance with BLUPF90. Contribution no. 21-296-J from the Kansas Agricultural Experiment Station.

\section{Authors' contributions}

$M B$ perfomred the analyses, wrote the main text and prepared the figures. $M R$, $\mathrm{JB}$, and RW supervised the work and assisted MB with statistical analyses. DG and $\mathrm{AH}$ provided reproductive physiology and semen collection procedures expertise. All authors read and approved the final manuscript.

\section{Funding}

Not applicable.

\section{Availability of data and materials}

The data that support the findings of this study are available from the American Angus Association and corresponding Al collection centers but restrictions apply to the availability of these data, which were used under license for the current study, and so are not publicly available. Data are available from the original data providers and/or the authors upon reasonable request and only with written permission of the original data providers and only in cases where this is consistent with the rules set forth in the data transfer agreement signed by both parties.

\section{Declarations}

\section{Ethics approval and consent to participate}

Study was undertaken with data owned by the American Angus Association and they consented to the work included in the manuscript.

\section{Consent for publication}

Not applicable.

\section{Competing interests}

The authors declare that they have no competing interests.

Received: 3 May 2021 Accepted: 13 December 2021

Published online: 23 January 2022

\section{References}

1. Bull management to maximize sperm output. In: Proceedings of the Sixth Technical Conference on Artificial Insemination and Reproduction. Milwaukie; 1976. p. 1-10. https://agris.fao.org/agris-search/search.do? recordID $=$ US201303059742. AGRICULTURAL SCIENCE AND TECHNOLOGY INFORMATION. agris.fao.org.

2. Chenoweth PJ. Sexual behavior of the bull: a review. J of Dairy Sci. 1983;66:173-9. https://doi.org/10.3168/jds.S0022-0302(83)81770-6.

3. Fuerst-Waltl B, Schwarzenbacher H, Perner C, Solkner J. Effects of age and environmental factors on semen production and semen quality of Austrian Simmental bulls. Anim. Repro. Sci. 2006;95:27-37. https://doi. org/10.1016/j.anireprosci.2005.09.002.

4. Brito LC, Barth AD, Rawlings NC, Wilde RE, Crews DH Jr, Mire PS, et al. Effect of improved nutrition during calfhood on serum metabolic hormones, gonadotropins, and testosterone concentrations, and on testicular development in bulls. Domestic Anim Endocrinol. 2006;33:460-9. https://doi.org/10.1016/j.domaniend.2006.09.004.

5. Coulter GH, Cook RB, Kastelic JP. Effects of dietary energy on scrotal surface temperature, seminal quality, and sperm production in young beef bulls. J Anim Sci. 1997;75:1048-52. https://doi.org/10.2527/1997.75410 48x.

6. Corbet NJ, Burns DM, Johnston DJ, Wolcott ML, Corbet DH, Venus BK, et al. Male traits and herd reproductive capability in tropical beef cattle. 2 . Genetic parameters of bull traits. Anim Prod Sci. 2013;53:101-13. https:// doi.org/10.1071/AN12163.

7. Huang JZ, Li Q, Hou Q, Wang C, Li J, et al. Solexa sequencing of novel and differentially expressed microRNAs in testicular and ovarian tissues in Holstein cattle. Int J Biol Sci. 2011;7:1016-26. https://doi.org/10.7150/ ijbs.7.1016.

8. Taylor JF, Schnabel RD, Sutovsky P. Identification of genomic variants causing sperm abnormalities and reduced male fertility. Anim Repro Sci. 2018;194:57-62.

9. Ben Jemaa S, Fritz S, Guillaume F, Druet T, Denis C, Eggen A, et al. Detection of quantitative trait loci affecting non-return rate in French dairy cattle. J Anim Breed Genet. 2008;125:280-8. https://doi.org/10.1111/j. 1439-0388.2008.00744.x.

10. Ron W, Feldmesser E, Golik M, Tager-Cohen I, Kliger D, Reiss V, et al. A complete genome scan of the Israeli Holstein population for quantitative trait loci by a daughter design. J Dairy Sci. 2004;2:476-90. https://doi.org/ 10.3168/jds.S0022-0302(04)73187-2.

11. Hoglund JK, Buitenhuis AJ, Guldbrandtsen B, Su G, Thomsen B, Lund MS. Overlapping chromosomal regions for fertility traits and production traits in the Danish Holstein population. J Dairy Sci. 2009;92:5712-9. https://doi. org/10.3168/jds.2008-1964.

12. Druet T, Fritz S, Sellem E, Basso B, Gerard O, Salas-Cortes L, et al. Estimation of genetic parameters and genome scan for 15 semen characteristics traits of Holstein bulls. J Anim Breed Genet. 2009;126:269-77. https:// doi.org/10.1111/j.1439-0388.2008.00788.x.

13. Costa A, Schwarzenbacher H, Mészáros G, Fuerst-Waltl B, Fuerst C, Sölkner J, et al. On the genomic regions associated with milk lactose in Fleckvieh cattle. J Dairy Sci. 2019. https://doi.org/10.3168/jds.2019-16663.

14. Bouwman AC, Visker MH, van Arendonk JA, Bovenhuis H. Genomic regions associated with bovine milk fatty acids in both summer and winter milk samples. BMC Genet. 2012;13:93.

15. Zhang Z, Kargo M, Liu A, Thomasen JR, Pan Y, Su G. Genotype-byenviroment interaction of fertility traits in Danish Holstein cattle using a single-step genomic reaction norm model. Heredity. 2019;123:202-14. https://doi.org/10.1038/s41437-019-0192-4. 
16. Hering DM, Olenski K, Kaminski S. Genome-wide association study for poor sperm motility in Holstein-Friesian bulls. Anim Reprod Sci. 2014;146:89-97. https://doi.org/10.1016/j.anireprosci.2014.01.012.

17. Jiang J, Ma L, Prakapenka D, VanRaden PM, Cole JB, Da Y. A large-scale genome-wide association study in U.S. Holstein cattle. Front Genet. 2019;10:412. https://doi.org/10.3389/fgene.2019.00412.

18. Westfalewicz B, Dietrich MA, Mostek A, Partyka A, Bielas W, Nizanski W, et al. Identification and functional analysis of bull (Bos taurus) cauda epididymal fluid proteome. J Dairy Sci. 2017;100:6707-19. https://doi.org/ 10.3168/jds.2016-12526.

19. Handrow RR, Lenz RW, Ax RL. Structural comparisons among glycosaminoglycans to promote an acrosome reaction in bovine spermatozoa. Biochem Biophys Res Commun. 1982;107:1326. https://doi.org/10.1016/ S0006-291X(82)80143-5.

20. Lane ME, Therien I, Moreau R, Manjunath P. Heparin and high density lipoprotein mediate bovine sperm capacitation by different mechanisms. Biol Reprod. 1999;60:169-75. https://doi.org/10.1095/biolreprod60.1.169.

21. Frischknecht M, Bapst B, Seefried FR, Signer-Hasler H, Garrick D, Stricker C, et al. Genome-wide association studies of fertility and calving traits in Brown Swiss cattle using imputed whole-genome sequences. BMC Genomics. 2017;18:910. https://doi.org/10.1186/s12864-017-4308-z.

22. Cole JB, Wiggans GR, Ma L, Sonstegard TS, Lawlor TJ Jr, Crooker BA, et al. Genome-wide association analysis of thirty one production, health, reproduction and body conformation traits in contemporary U.S. Holstein cows. BMC Genomics. 2011;12:408. https://doi.org/10.1186/ 1471-2164-12-408.

23. Schnabel RD, Sonstegard TS, Taylor JF, Ashwell MS. Whole-genome scan to detect QTL for milk production, conformation, fertility and functional traits in two US Holstein families. Anim Genet. 2005;36:408-16. https:// doi.org/10.1111/j.1365-2052.2005.01337.x.

24. Cai Z, Guldbrandtsen B, Lund MS, Sahana G. Prioritizing candidate genes for fertility in dairy cows using gene-based analysis, functional annotation and differential gene expression. BMC Genomics. 2019;20:255 doi: s12864-019-5638-9.

25. Sweett H, Fonseca PAS, Suárez-Vega A, Livernois A, Miglior F, Canovas A. Genome-wide association study to identify genomic regions and positional candidate genes associated with male fertility in beef cattle. Sci Rep. 2020;10:20102. https://doi.org/10.1038/s41598-020-75758-3.

26. Butler ML, Hartman AR, Bormann JM, Weaber RL, Grieger DM, Rolf MM. Genetic parameter estimation for beef bull semen attributes. J Anim Sci. 2021;99(2):skab013. https://doi.org/10.1093/jas/skab013. https:// pubmed.ncbi.nlm.nih.gov/33453111/.

27. Misztal I, Tsuruta S, Laurenco DAL, Masuda Y, Aguilar I, Legarra A, et al. Manual for BLUPF90 family programs: University of Georgia; 2016. http:// nce.ads.uga.edu/wiki/doku.php?id=documentation

28. Wang H, Misztal I, Aguilar I, Legarra A, Muir WM. Genome-wide association mapping including phenotypes from relatives without genotypes. Genet Res. 2012;94:73-83. https://doi.org/10.1017/S0016672312000274.

29. Masuda, Y. Introduction to BLUPF90 suite programs Concise version. University of Georgia. April 2019. Pp. 180-181. http://nce.ads.uga.edu/ wiki/lib/exe/fetch.php?media=tutorial_blupf90.pdf. Revision History March 2018 (0.8.0): The first revision prepared for the summer course at UGA. Thanks to Andrés Legarra. April 2019 (0.9.0): Revised to correct some errors and typos. nce.ads.uga.edu.

30. Aguilar I, Legarra A, Cardoso F, Masuda Y, Lourenco D, Misztal I. Frequentist $\mathrm{p}$-values for large-scale-single step genome-wide association, with an application to birth weight in American Angus cattle. Genet Sel Evol. 2019:511-8.

31. Garrick DJ. Equivalent mixed model equations for genomic selection. J Anim Sci. 2007:85:376

32. Yin L. Circular manhattan plot. R package version 3.6.0. 2020. https:// github.com/YinLiLin/R-CMplot

33. Storey JD, Bass AJ, Dabney A, Robinson D. qvalue: Q-value estimation for false discovery rate control. R package version 2.20.0. 2020. http://github. com/jdstorey/qvalue.

34. McKay SD, Schnabel RD, Murdoch BM, Matukumalli LK, Aerts J, Coppieters W, et al. Whole genome linkage disequilibrium maps in cattle. BMC Genet. 2007:8:74

35. Hu Z, Park CA, Reecey JM. Building a livestock genetic and genomic information knowledgebase through integrative developments of animal
ATLdb and CorrDB. Nucleic Acids Res. 2019;47:D701-10. https://doi.org/ 10.1093/nar/gky1084.

36. The UniProt Consortium. UniProt: a worldwide hub of protein knowledge Nucleic Acids Res. 2019;47:D506-15

37. Huang DW, Sherman BT, Lempicki LA. Bioinformatics enrichment tools:paths toward the comprehensive functional analysis of large gene lists. Nucleic Acids Res. 2009;37:1-13. https://doi.org/10.1093/nar/gkn923.

38. Huang W, Kirkpatrick BW, Rosa GJ, Khatib H. A genome-wide association study using selective DNA pooling identifies candidate markers for fertility in Holstein cattle. Anim Genet. 2009;41:570-8. https://doi.org/10. 1111/j.1365-2052.2010.02046.x.

39. Kiser JN, Keuter EM, Seabury CM, Neupane M, Moraes JGN, Dalton J, et al. Validation of 46 loci associated with female fertility traits in cattle. BMC Genomics. 2019;20:576. https://doi.org/10.1186/s12864-019-5935-3.

40. Boichard D, Grohs C, Bourgeois F, Cerqueira F, Faugeras R, Neau A, et al. Detection of genes influencing economic traits in three French dairy cattle breeds. Genetics. 2003;1:77-101. https://doi.org/10.1186/ 1297-9686-35-1-77.

41. Schrooten C, Bink MCAM, Bovenhuis H. Whole genome scan to detect chromosomal regions affecting multiple traits in dairy cattle. J Dairy Sci. 2004;87:3550-60. https://doi.org/10.3168/jds.S0022-0302(04)73492-X.

42. Hawthorne SK, Goodarzi G, Bagarova J, Gallant KE, Busanelli RR, Olend WJ, et al. Comparative genomics of the sperm mitochondria-associated cysteine-rich protein gene. Genomics. 2006;87:382-91. https://doi.org/10. 1016/j.ygeno.2005.09.010

43. Ashwell MS, Heyen DW, Weller JI, Ron M, Sonstegard TS, Van Tassell CS, et al. Detection of quantitative trait loci influencing conformation traits and calving ease in Holstein-Friesian cattle. J Dairy Sci. 2005;88:4111-9. https://doi.org/10.3168/jds.S0022-0302(05)73095-2.

44. Senger PL. Pathways to pregnancy and parturition. 3rd ed. Redmon, Oregon: Current Conceptions, Inc.; 2012

45. Melo TP, Fortes MRS, Bresolin T, Mota LFM, Albuquerque LG, Carvalheiro R. Multitrait meta-analysis identified genomic regions associated with sexual precocity in tropical beef cattle. J Anim Sci. 2018;96:4087-99. https://doi.org/10.1093/jas/sky289.

46. McClure MC, Morsci NS, Schnabel RD, Kim JW, Yao P, Rolf MM, et al. A genome scan for quantitative trait loci influencing carcass, post-natal growth and reproductive traits in commercial Angus cattle. Anim Genet. 2010;4:597-607. https://doi.org/10.1111/j.1365-2052.2010.02063.x.

47. Meyer K, Hammond K, Mackinnon MJ, Parnell PF. Estimates of covariances between reproduction and growth in Australian beef cattle. J Anim Sci. 1991:69:3533-43.

48. Neves H, Vargas G, Brito LF, Schenkel FS, Albuquerque LG, Carvalheiro R. Genetics and genomic analyses of testicular hypoplasia in Nellore cattle. PLoS One. 2019;14:e0211159. https://doi.org/10.1371/journal.pone.02111 59.

49. Schulman NF, Sahana G, Iso-Touru T, McKay SD, Schnabel RD, Lund MS, et al. Mapping of fertility traits in Finnish Ayrshire by genome-wide association analysis. Anim Genet. 2011;42:263-9. https://doi.org/10.1111/j. 1365-2052.2010.02149.x.

50. Pimentel EC, Bauersachs S, Tietze M, Simianer H, Tetens J, Thaller G, et al. Exploration of relationships between production and fertility traits in dairy cattle via association studies of SNPs within candidate genes derived by expression profiling. Anim Genet. 2011;42:251-62. https://doi. org/10.1111/j.1365-2052.2010.02148.x.

51. Blaschek M, Kaya A, Zwald N, Memili E, Kirkpatrick BW. A whole-genome association analysis of noncompensatory fertility in Holstein bulls. J Dairy Sci. 2011;94:4695-9. https://doi.org/10.3168/jds.2010-3728.

52. DeJarnette JM, Marshal CE, Lenz RW, Monke DR, Ayars WH, Sattler CG. Sustaining the fertility of artificially inseminated dairy cattle: the role of the artificial insemination industry. J Dairy Sci. 2004;87(E. Suppl):E93E104. https://doi.org/10.3168/jds.S0022-0302(04)70065-X.

53. Hansen M. Genetic investigations on male and female fertility in cattle. Livest Sci. 1979:6:325-34.

54. Syrstad O. Selection for fertility in the basis of Al data. Livest Prod Sci. 1981;8:247-52.

55. Christmas RA, Moser DW, Spire MF, Sargeant JM, Tucker SK. Genetic relationships among breeding soundness traits in yearling bulls. In: Cattlemen's Day. Manhattan; 2001. p. 1-3. https://newprairiepress.org/cgi/ viewcontent.cgi?referer=https://www.google.com/\&httpsredir=1\&artic $l e=1712 \&$ context $=$ kaesrr 
56. Zhou Y, Connor EE, Wiggans GR, Lu Y, Tempelman RJ, Schroeder SG, et al. Genome-wide copy number variant analysis reveals variants associated with 10 diverse production traits in Holstein cattle. BMC Genomics. 2018;19:314. https://doi.org/10.1186/s12864-018-4699-5.

57. Deprez P, Inestrosa NC, Krejci E. Two different heparin-binding domains in th triple-helical domain of ColA, the collegen tail subunit of synaptic acetylcholinesterase. J Bio Chem. 2003;278:23233-42. https://doi.org/10 1074/jbc.M301384200.

58. Huszar G, Patrizio P, Vigue L, Willets M, Wilker C, Adhoot D, et al. Cytoplasmic extrusion and the switch from creatine kinase $B$ to $M$ isoform are completed by commencement of epididymal transport in human and stallion spermatozoa. J Androl. 1998;19:1 1-20. https://doi.org/10.1002/j. 1939-4640.1998.tb02465.x.

59. Doran J, Walters C, Kyle V, Wooding P, Hammett-Burke R, College B.

Mfsd14a (Hiat1) gene disruption causes globozoospermia and infertility in male mice. Repro. 2016;152:91-9. https://doi.org/10.1530/REP-15-0557.

\section{Publisher's Note}

Springer Nature remains neutral with regard to jurisdictional claims in published maps and institutional affiliations.

- fast, convenient online submission

- thorough peer review by experienced researchers in your field

- rapid publication on acceptance

- support for research data, including large and complex data types

- gold Open Access which fosters wider collaboration and increased citations

- maximum visibility for your research: over $100 \mathrm{M}$ website views per year

At BMC, research is always in progress.

Learn more biomedcentral.com/submissions 